\title{
The future of pediatric US
}

\author{
Brian D. Coley
}

Received: 22 December 2010 /Revised: 4 January 2011 / Accepted: 13 January 2011

(C) Springer-Verlag 2011

\begin{abstract}
Despite advances in other modalities, US remains an essential technology in pediatric imaging. Improvements in technology provide increasingly detailed anatomical images, and new techniques show promise in providing novel anatomical as well as physiological and structural information. Other specialties are recognizing the value of US to their patients and are increasingly performing their own examinations. Economic and social factors are also influencing the development and use of US. This review will evaluate many of these issues and demonstrate that the future of pediatric US is bright and that pediatric radiologists can and should continue to be leaders in its use and development.
\end{abstract}

Keywords US · Pediatric $\cdot$ Point-of-care $\cdot$ Technology

\section{Introduction}

US is the most widely used advanced imaging modality in the world [1]. Although our adult-imaging colleagues appreciate the value of US in some circumstances, in many radiology departments in the United States, US is an orphan modality with little radiologist involvement or support. Regrettably, many radiologists in the United States are poor sonologists, relying solely upon the opinions of the

Disclaimer Dr. Coley has no financial interests, investigational or offlabel uses to disclose.

B. D. Coley $(\bowtie)$

Department of Radiology, Nationwide Children's Hospital,

700 Children's Drive,

Columbus, OH 43205, USA

e-mail: brian.coley@nationwidechildrens.org sonographers who perform the examinations to do the work of making diagnoses. In countries without sonographers, where the physician performs the US examination, the power and utility of US are better appreciated and the understanding of US images and diagnoses stronger. Pediatric radiologists are, in general, more familiar with US and its considerable diagnostic capabilities. Having generally smaller bodies with lesser amounts of fat, children make ideal US subjects and allow the use of higher-frequency transducers that subsequently produce images with greater spatial and contrast resolution than are possible in most adults. And of course, what US commentary would be complete without mentioning the obvious lack of radiation exposure to the patient.

Despite such obvious strengths and benefits, and the remarkable improvement in US technology and image quality, US has received comparatively little attention in the imaging world during the last decade. CT and MR have been the focus of most imaging research and clinical evaluation, and this is not without reason. Both modalities have broad clinical applications, produce precise anatomical images and can provide functional and physiological information as well. That said, US is still the first-line imaging test for many clinical indications and has the potential to be definitive and the only imaging test needed for many pediatric patients. But with the advances in other areas of imaging, will US remain a viable and important part of pediatric health care? The answer is unequivocally "yes," for a variety of reasons to be discussed below. This is the encouraging part for those of us with a particular interest in US imaging. Will radiologists be the ones performing studies in the future or leading the way in the development of new techniques and applications? That is less certain. Radiologists will have to be more involved if we are to remain relevant to pediatric sonography. 
What forces will make US relevant to future pediatric imaging (and, in fact, lead to its expansion)?

Concern over pediatric radiation exposure

Miniaturization and US availability

Utilization trends and point-of-care US

Economic drivers

Advances in US technology

Each of these areas could be the subject of lengthy papers, as they are each individually complex and multifaceted topics. However, I will attempt to outline briefly some of the key areas supporting my contention that the future of pediatric US is bright.

\section{Concern over pediatric radiation exposure}

The ECRI named radiation exposure from CT scans the No. 4 threat to patient safety in its November 2010 report [2]. Europe has long been a better steward of radiation dose and protection compared to the rest of the world, especially the United States, and US is often dismissed as an orphan modality in United States radiology training programs. Fortunately, US has always been a strong part of most pediatric radiology practices, but we, too, experienced the explosive growth in body $\mathrm{CT}$ imaging that occurred over the last two decades. In the United States, there were about 70 million, and in the UK about 3 million, CT scans performed in 2009 [3], with estimates that $5 \%$ to $10 \%$ of these scans were performed for children [3]. In a recent article, Brenner [3] outlines efforts to reduce radiation dose that include the reduction in individual dose for each $\mathrm{CT}$ exam, the reduction in the number of exams through the use of clinical decision rules, and the use of alternative nonradiation imaging modalities.

In the United States, CT is frequently the study of choice when imaging the abdomen and pelvis. Thanks to articles in 2001 calling attention to frequently inappropriate dose regimens in most pediatric $\mathrm{CT}$ studies and the possible consequences $[4,5]$, and the important work of the Alliance for Radiation Protection in Pediatric Imaging's Image Gently campaign, pediatric CT use is dropping. At my own institution, the rate of CT scan growth has steadily decreased since 2000, with marked negative growth during the last 2 years (Fig. 1). In 2003, CT accounted for $13.4 \%$ of all of our imaging studies, whereas in 2010 it accounted for only $8.8 \%$; other pediatric institutions are experiencing similar trends (Society of Chairmen of Radiology in Children's Hospitals practice survey, 2010, personal communication with P. J. Strouse, C.S. Mott Children's Hospital, Ann Arbor).

So what has happened to all of those patients who used to get CT scans? Certainly some patients were watched

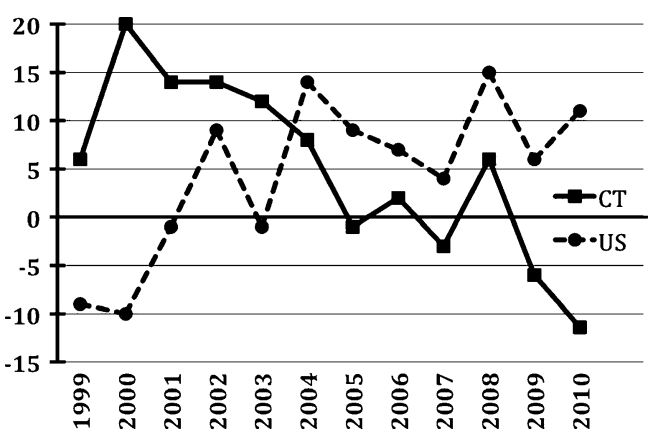

Fig. 1 Percentage change from previous year of number of CT and US studies performed at Nationwide Children's Hospital, Columbus, $\mathrm{OH}$

longer, or had a physical exam by a more experienced clinician, rather than being run through the CT scanner. Others, however, had a US (or perhaps an MR) exam performed instead. During the same time period of CT scan use decline, we have seen a corresponding increase in US use at my institution (Fig. 1) above the growth in overall departmental imaging volume. Some of this increase can be credited to our radiologists being more focused on diverting patients from CT when appropriate, but much of the credit must be given to our clinicians becoming more cognizant about radiation issues when deciding to image. Our emergency department is increasingly using sonography as the initial imaging examination in cases of acute appendicitis, with $\mathrm{CT}$ being reserved for complex or indeterminate cases, a strategy that many authors have shown can markedly reduce CT use without negatively impacting patient outcomes $[6,7]$.

Clearly, there are geographical variations in practice. Even within North America there are considerable differences in the utilization of CT and US. Using data summing the total number of plain radiographic, CT, US, MR and nuclear medicine examinations from the 2010 SCORCH survey (P. J. Strouse, personal communication), CT scans accounted for $9.8 \%$ of studies in the United States but only $6.1 \%$ of studies in Canada. In the United States, US accounted for $11.3 \%$ of studies, while in Canada US accounted for $20.8 \%$. Consider also the difference in imaging guidelines for oncology follow-up between North America and Europe. Staging and surveillance of most pediatric cancers in the United States rely primarily on contrast-enhanced CT, whereas in Europe imaging of the abdomen and pelvis relies primarily on MRI and US [8]. Studies in process with the American College of Radiology Imaging Network (ACRIN) are evaluating the use of whole-body MRI in staging pediatric malignancy [9], and other researchers are pursuing similar studies in an effort to minimize imaging-related radiation exposure $[10,11]$. In my opinion, given the improvements in US technology and the quality of anatomical and physiological information 
obtainable, and the advancement of MR imaging with faster sequences and more focused protocols, within 10 years almost all imaging of the abdomen and pelvis will be performed with US or MR.

\section{Miniaturization and availability}

The miniaturization of US units has been remarkable during the last decade. While most radiologists would not have given these units a second glance in the past, image quality and functionality of many of these compact US units rival that found in traditional systems. As reported in "The World Market for Ultrasound Imaging Equipment - 2010 Edition" [12] the market for compact US was $\$ 810.9$ million in 2009, a $15.3 \%$ increase in revenue from 2007. The 2014 forecast is for the compact US market to be worth $\$ 1.25$ billion, a 55\% increase over 2009. Radiologists account for a tiny part of the growth in this market. These compact machines have captured the attention of nonradiology physicians increasingly performing point-of-care US in nontraditional settings. The development of new applications (especially in procedural guidance), and the expansion into underserved regions because of diminished costs have also fueled the expansion of this market [13-15]. These new users are attracted to the speed, flexibility, sufficient (and often excellent) image quality, and lower costs of purchase and maintenance of these compact systems. This economic and sales forecasting report also discusses the US equipment market as a whole, and notes: "Compact ultrasound will not replace specialized cart-based systems in radiology" [12].

This last statement is important, as it applies not only to the machines, but also to the people using them. Compact US and the expansion into the hands of physicians outside the usual imaging-based specialties will not erode the importance of the expert diagnostic sonologist. Like others, I don't see the pie being divided into smaller pieces for each subspecialty interest group; I see the pie getting bigger [16]. This is borne out in the increasing volume of US examinations in pediatric radiology departments. In fact, when emergency departments start using US, radiology departments might actually see a short-term increase in imaging referrals [17].

As physicians concerned with improving pediatric care and who recognize the potential diagnostic benefits of US, we should be pleased with this trend. However, it is difficult not to feel some threat from groups wanting to take over some of the duties in which we have spent so long developing expertise. Clearly, there are issues of education and competency to ensure that those performing US are adequately trained, and decisions on whether US is truly helpful in various clinical scenarios. As far as the further expansion of compact US and its potential impact on traditional radiology practices: there's no stopping it, so we in radiology need to get over it and determine how best to use our expertise in this new imaging landscape. We shouldn't be petulant and refuse to cooperate with our clinical colleagues who express a genuine interest (beyond concerns of financial gain) in developing uses for US in their practices. If anything, we should be involved to ensure that if US is being used, it is being used appropriately and the people using it have the skills needed to help (and not hurt) patients. This can be hard to do, as there can be financial, political and prestige stakes for all parties. However, if these can be overcome, the cooperation among specialties can be very fruitful, as has been shown by consensus statements and training guidelines developed by societies such as the American Institute for Ultrasound in Medicine, which is composed of multiple medical specialties interested in improving patient care through the best practice use of sonography.

\section{Utilization trends and point-of-care US}

As reported by Ridley [18], according to United States Medicare data, the growth rate of all imaging modalities declined $66 \%$ for the period 2005-2009 compared with 1998-2005. Non-cardiac US, however, showed the smallest decline (40\%, compared to MR at $93 \%)$ and thus showed a relative share increase compared to other modalities. As shown in other studies, growth rates remain higher among nonradiologists when compared to radiologists [19].

The usual argument by radiologists for the increased use of imaging by nonradiologists (both in per patient exams ordered and in rate of growth of all imaging services) centers on self-referral and financial incentives rather than interests of patient care. Some of this is certainly true. When imaging is readily available and performed by specialty-trained radiologists, the incentives around providing imaging services not overseen or interpreted by imaging experts can appropriately be viewed with suspicion.

But that is only part of the story, especially with US. There is solid evidence that the use of US guidance in the performance of procedures improves procedural success and minimizes complications. In the United States, the Institute of Medicine and the Agency for Healthcare Quality and Research listed the implementation of USguided central venous access over traditional blind access as one of the top 10 changes that could most improve patient care [20]. In other scenarios, point-of-care US with very focused problem-oriented examinations is being shown to expedite diagnostic evaluations and interventional 
procedures and to improve patient care [13, 15, 21]. Ignoring for a moment the issues of money, politics, egos, resource duplication and utilization, performance quality and documentation, it is hard not to be in favor of the spread of US out of the hands of the traditional imaging experts for certain indications. Is this necessarily a bad thing? I don't think so. Would you want your child in the intensive care unit to have a central line placed with or without US guidance? As one of our institution's interventional radiologists, I cannot possibly be present in a timely manner for every central line, thoracentesis or nerve block done in my hospital, nor should critically ill patients have to wait for me. Further, I do not want to do all of these procedures. It is not an optimal use of my time and US expertise. However, it would be irresponsible to capitulate and allow just anyone with a desire to scan a patient the privilege to do so. As imaging experts who care about the proper and accurate imaging of children, we need to remain involved in the training of new user groups and be advocates for appropriate imaging within our countries, societies and institutions. It should not be "us" versus "them," but rather "we" striving to take the best care of our patients.

Although data suggest that certain point-of-care US studies and procedures can be adequately performed by clinicians, not all clinicians will feel the need to adopt these procedures, particularly if clinically relevant imaging and procedural expertise is readily available elsewhere. Just as I don't want or need to perform every vascular access procedure, my emergency department colleagues really don't want to (or at least they shouldn't want to) perform every US study needed in their department. The main performance metrics that my emergency department is held to are speed of patient throughput first, and accuracy of diagnosis second. Imaging is an integral part of many emergency department diagnoses, and the increased use of imaging services has been shown to increase emergency department length of stay [22]; emergency departments need to integrate imaging efficiently into their patient evaluations. If our radiology department is available with our US expertise, it is more expedient and accurate for my emergency department colleagues to have us image the patient while they go on and use their expertise to see more patients.

So what does this all mean to us as pediatric imagers? We have to understand what our clinical colleagues need. We have to be available when the clinicians and their patients need imaging. We have to stay engaged and involved in the patient care process. We have to remain involved in the process of training and performance competency for those clinicians performing point-of-care studies. If we do these things, then we will remain relevant to the practice of sonography and will remain valuable to our patients and clinicians.

\section{Economic drivers}

Spending on health care consumed $10.2 \%$ of global gross domestic product (GDP) in 2004, ranging from $2 \%$ in the Congo to $15.2 \%$ in the United States [23]. Despite a downturn in the global economy, the Centers for Medicare and Medicaid Services state that health care spending in the United States grew to $17.3 \%$ of the U.S. GDP in 2009 [24]. The complexities of health care costs form the basis of entire careers and are thus too much to cover completely in this short paper. However, imaging costs in one study were found to account for about $10 \%$ of health care costs between 1996 and 2002 [25]. A more recent report found that the volume of imaging services among Medicare patients increased at double the rate of other physician services [26]. One's opinion on whether rising imaging expenditures are reasonable and lead to decreased overall health care costs and improved outcomes [27-29], represent irresponsible self-referral $[19,30]$, or are a by-product of defensive medicine practices $[30,31]$ depends upon one's perspective.

Regardless of the actual numbers, there is no doubt that imaging costs are significant, and given the visibility of imaging technology, these costs are under increasing scrutiny. With increasing federal and state deficits, healthcare expenditures are being continually re-evaluated. The pressure to cut costs is tremendous. As money for patient care becomes more scarce, reimbursement for imaging procedures will no doubt decrease, and the demand for proving patient care value for these imaging dollars will increase. Actual imaging costs are difficult to discern, but there is no argument that US is less expensive than CT. The return on investment even for premium US equipment is less than 1 year, making equipment purchases easier to justify. In this environment, US becomes a very attractive modality, particularly in pediatric imaging, where US can often provide definitive answers and not just serve as a screening modality (and may, in part, explain the previously mentioned greater use in Canada of US compared with CT).

\section{Advances in US technology}

Aside from the application of US to new and novel clinical applications, there have been (and continue to be) tremendous advances in the technology of US and in the information it provides to us as clinicians and researchers. Understanding these new technologies will help us to take 
better advantage of them and to improve the care of our patients.

\section{Transducers}

Much of the improvement in US parallels other advances in computer technology that enable the processing of more and more information in shorter and shorter periods of time in smaller and smaller devices. With the capability to process more information, manufacturers have developed increasingly sophisticated transducers to supply more information to these processors. Transducers now have thousands of elements coupled to electronic beam formers that allow the transmittal of multiple angles of insonation. Coupled with various companies' proprietary soundencoding technologies, these advances allow images of previously unimagined high spatial, temporal and contrast resolution [32]. More detailed information on specific transducer and sound pulse technologies can be found in an excellent recent review by Dumitriu et al. [32].

\section{Image display}

During the development of US, the introduction of the compound B-scan was considered a great leap forward, as it allowed the display of a large "slice" of anatomy. Real-time sonography provides much greater anatomical detail but at the cost of a much smaller window into the body. Extended field-of-view techniques now found in most equipment give the best of both worlds. These images allow visualization of large areas of anatomy for more complete visualization and understanding of anatomical relationships, albeit with somewhat reduced resolution. This can certainly make it easier for us to demonstrate structures for our clinical colleagues, but these images can also improve our understanding and make our diagnoses more accurate $[33,34]$.

\section{3-D US and volume data acquisition}

The idea of 3-D US dates to the 1960s, but the aforementioned advancements in transducer and computing technology have recently made it a practical reality available outside the research lab [35]. Sometimes still viewed as a novelty or marketing toy, 3-D US has proved valuable in many diagnostic applications, particularly in obstetrics and gynecology [36, 37]. The value has come in the ability to visualize structures in three dimensions, visualization in reconstructed planes not otherwise obtainable or viewable with conventional two-dimensional acquisitions, and improved appreciation of 2-D images. The utility and role of 3-D US in pediatric diagnosis is unclear, but there has been very interesting work in the area of neurosonology [38, 39], the genitourinary tract $[39,40]$, the musculoskeletal system [41] and other areas. With improvements in offline processing and visualization of 3-D data sets that speed image evaluation and make work flow more practical in busy departments, 3-D imaging should find its niche in pediatric US during the next several years.

The development of volume data acquisition capabilities that allow 3-D imaging has also brought the potential for changes in the way we perform US examinations. The person performing the US exam can now acquire a series of volumes from particular anatomical regions to study later and reconstruct in any way desired. In our department, a typical renal US examination consists of about 30 still images, and often a couple of cine sweeps through the kidneys. Consider now performing just three volume acquisitions - one for each kidney and one for the bladder. These three data sets could then be evaluated and displayed later in any plane desired. Just as we face in complex CT and MR studies, the question of who takes the time to perform these offline image analyses (technologists or physicians) and how this is to be incorporated efficiently into a busy practice remains unsettled. However, some authors have reported increased efficiencies for this type of practice, although one must first be very facile with the technology $[42,43]$. In countries using sonographers to acquire images, this raises issues of what skills are needed to perform exams. The training of someone to get a volume of a kidney is less than that of someone who has to capture the perfect renal image in a single still frame. The ability of someone with less training to capture volumes that someone more expert could subsequently evaluate might allow US studies to be performed remotely and thus be accessible by more patients [44]. Being someone who works with sonographers, I have mixed feelings about this, because a skilled sonographer is a second set of eyes and hands and a colleague who often detects subtleties on a study needing further evaluation and hears aspects of the history from patients and parents that can direct our examination.

\section{Contrast-enhanced US}

US, CT and MR provide both similar and distinctive anatomical information depending upon the application. One thing that currently sets CT and MR apart is the additional information provided by intravascular contrast material. Although US contrast agents exist, they are generally not approved for pediatric use, and in the United States are not approved at all for general imaging purposes. The use of these agents does raise concerns about possible bioeffects as these materials interact with US that will require us to pay greater attention to our US energy outputs 
[45]. Pharmacologically, however, these agents have been shown to be quite safe with a very low incidence of side effects and allergic reactions [32, 46]

The use of US contrast agents in pediatrics will require a shift of attitude in how we perceive the use of sonography in our practices. We like sonography because it's noninvasive and relatively fast; we do not have to take the time to start an intravenous line, administer a contrast agent (that might not be reimbursable), or perform pre- and postcontrast imaging. However, there is no doubt that US contrast agents can increase the sensitivity and specificity of US diagnoses. The temporal resolution of contrast kinetics with US has been shown to be superior to that of both $\mathrm{CT}$ and MR and has proved to provide markedly improved accuracy in the characterization of liver masses in adults [47, 48]. Contrast-enhanced US can improve the detection and evaluation of renal abnormalities, venous thromboses and organ perfusion and can aid in the assessment of neoplasms and their response to treatment [48]. A nonvascular application of US contrast agent that has demonstrated value is in the evaluation for vesicoureteral reflux [49]. Newer applications combine US contrast agents and molecular imaging; by attaching specific receptors to the outer contrast bubble shell, the contrast agent can be made to target specific cells or structures. This opens the possibility of increasing diagnostic sensitivity and specificity, along with the ability to deliver specific medications and therapies within these contrast microbubbles that can then be burst by US when accumulated at the appropriate target $[50,51]$. The use of these agents would require more effort on our behalf, but the potential benefit to our patients could be great.

\section{Tissue characterization}

The ability to noninvasively characterize tissue structure, composition and physiology is a goal of all advanced imaging technologies. For US, basic changes in tissue echogenicity and vascular impedance or perfusion with Doppler are methods of tissue characterization, but in general provide only gross and nonspecific information about the structure imaged. US contrast agents can improve accuracy about focal lesions but still do not provide specific detail about structure. Interesting work on speckle patterns and the quantitation of structural elements from unprocessed US signals has shown that these techniques can provide additional information [52, 53], but for now these remain primarily research tools and are not applicable in most US practices.

One technique that has begun to emerge as clinically practical is elastography or strain imaging. The technique differs among manufacturers, and the physical principles are well described elsewhere [53, 54], but the basic concept is the measurement of tissue stiffness. By assessing how tissue moves in response to either direct pressure or pressure supplied by a US pulse, one can infer its stiffness. This has been used in the breast and thyroid to try to better characterize benign and malignant lesions (malignancies tend to be stiffer) with reasonable success $[55,56]$. More recently, the evaluation of liver stiffness, and by inference the degree of fibrosis, has been the subject of many adult and a few pediatric papers [57-60]. The increase in liver disease in both adults and children makes it very important to develop a noninvasive method of detecting and following hepatic fibrosis without the need for biopsy. Recent advances allow liver strain imaging to be done rapidly without the need for sophisticated offline data processing. This imaging yields reproducible quantitative results that should allow longitudinal patient evaluation. These techniques have been applied to other organ systems as well as echocardiography, and I believe they will ultimately find utility in routine practice.

\section{Therapeutic US}

In the last several decades, US has been a diagnostic modality, although early in its history (and in some recent discredited treatments) US was primarily used in musculoskeletal therapies. More recently, developments in US technology are leading to exciting therapeutic possibilities for sonography.

High-intensity focused US (HIFU) takes advantage of the deposition of heat caused by US interaction with tissue. Like other technologies that deposit energy within tissue (such as radiofrequency ablation), this can be used to produce local tissue death and thus become a method of tissue ablation treatment. HIFU has the advantage over other energy deposition methods in that the sound can be directed through the skin, without the need for percutaneous placement of needles or probes. HIFU has been shown to successfully treat liver and kidney tumors, uterine fibroids, prostate tumors and skeletal lesions and to stop solid organ bleeding [61-65]. Reports of its use in children are limited, but HIFU promises to become an important part of treatment.

Another therapeutic use of US showing clinical potential is thrombolysis, often in conjunction with US contrast agents. Studies show that accelerated thrombolysis using low-frequency US might speed reperfusion and diminish complications from intravascular thrombolytics [66]. Another novel application of US is to increase the permeability of the blood-brain barrier to facilitate the delivery of medications [67]. Similarly, US contrast agents containing gene vectors have been used to target gene therapy delivery [68]; by destroying these microbubbles with higher-energy US pulses, the delivery vectors can be released at precisely 
desired locations. Clearly these applications of US are beyond the current role of diagnostic radiology. Nonetheless, they reinforce the importance of US to pediatric diagnosis and treatment.

\section{Conclusion}

In this overview, I hope that I've convinced you that the future of pediatric US is bright and promising. Many factors are involved in the success of any particular imaging modality. Some of these factors are under our personal control: our education and development of expertise, our involvement in research. Some of these factors can be influenced by our actions and behaviors: our availability and interaction with clinical colleagues, our involvement in societies and political organizations, our willingness to try new techniques. And some of these factors are often beyond our control: global economics, changes in political parties, societal attitudes. However, taken together all of these factors indicate that US should remain a strong and competitive imaging technology and that its benefits to the children that we care for will continue to expand and increase. US remains an exciting and advancing field, and despite increasing use by other specialties, radiology and radiologists can and should remain at its forefront.

\section{References}

1. Porter J, Jablon MS (2002) Musculoskeletal ultrasound. In: Weiner RS (ed) Pain management: a practical guide for clinicians. CRC Press, Boca Raton, p 1061

2. ECRI (2010) Top 10 health technology hazards for 2011. ECRI Institute. Available via https://www.ecri.org/Forms/Pages/ Top_10 Technologies.aspx. Accessed 26 Dec 2010

3. Brenner DJ (2010) Slowing the increase in the population dose resulting from CT scans. Radiation Res 174:809-815

4. Brenner DJ, Elliston CD, Hall EJ et al (2001) Estimated risks of radiation-induced fatal cancer from pediatric CT. AJR 176:289-296

5. Paterson A, Frush DP, Donnelly LF (2001) Helical CT of the body: are settings adjusted for pediatric patients? AJR 176:297-301

6. Ramarajan N, Krishnamoorthi R, Barth R et al (2009) An interdisciplinary initiative to reduce radiation exposure: evaluation of appendicitis in a pediatric emergency department with clinical assessment supported by a staged ultrasound and computed tomography pathway. Acad Emerg Med 16:1258-1265

7. Wan MJ, Krahn M, Ungar WJ et al (2009) Acute appendicitis in young children: cost-effectiveness of US versus CT in diagnosisa Markov decision analytic model. Radiology 250:378-386

8. Voss SD, Reaman GH, Kaste SC et al (2009) The ALARA concept in pediatric oncology. Pediatr Radiol 39:1142-1146

9. Siegel MJ (2010) Whole-body MRI in the evaluation of pediatric malignancies. Available via http://www.acrin.org/TabID/184/ Default.aspx. Accessed 26 Dec 2010

10. Darge K, Jaramillo D, Siegel MJ (2008) Whole-body MRI in children: current status and future applications. Eur J Radiol 68:289-298
11. Ley S, Ley-Zaporozhian J, Schenk JP (2009) Whole-body MRI in the pediatric patient. Eur J Radiol 70:442-451

12. (2010) InMedica predicts $\$ 1.25 \mathrm{Bn}$ market for compact ultrasound. TMCnet Available via http://www.tmenet.com/usubmit/2010/08/ 09/4947369.htm. Accessed 26 Dec 2010

13. Evans N, Gournay V, Cabanas F et al (2011) Point-of-care ultrasound in the neonatal intensive care unit: international perspectives. Sem Fetal Neonat Med 16:61-68

14. Harris RD, Marks WM (2009) Compact ultrasound for improving maternal and perinatal care in low-resource settings. J Ultrasound Med 28:1067-1076

15. Martin LD, Howell EE, Ziegelstein RC et al (2009) Hand-carried ultrasound performed by hospitalists: does it improve cardiac physical examination? Am J Med 122:35-41

16. Orenstein BW (2009) Ultrasound exams - bright future, but will it be in radiology? Radiol Today 10:12

17. Jacoby JL, Kasarda D, Melanson S et al (2006) Short- and long-term effects of emergency medicine sonography on formal sonography use: a decade of experience. J Ultrasound Med 25:233-236

18. Ridley EL (2010) Imaging utilization growth hits the brakes. AuntMinnie.com

19. Levin DC, Rao VM (2008) Turf wars in radiology: updated evidence on the relationship between self-referral and the overutilization of imaging. J Am Coll Radiol 5:806-810

20. Rothschild JM (2001) Chapter 21. Ultrasound guidance of central vein catheterization. Making health care safer: a critical analysis of patient safety practices. Evidence Report/Technology Assessment No. 43. Agency for Healthcare Research and Quality, Rockville, MD, USA pp 245-253

21. Dan D, Mingsong L, Jie T et al (2010) Ultrasonographic applications after mass casualty incident caused by Wenchuan earthquake. J Trauma 68:1417-1420

22. Herring A, Wilper A, Himmelstein DU et al (2009) Increasing length of stay among adult visits to U.S. emergency departments, 2001-2005. Acad Emerg Med 16:609-616

23. (2007) Almost half of all world health spending is in the United States. Progressive Policy Institute. Available via http://www. ppionline.org/ppi_ci.cfm?contentid $=254167 \& \mathrm{knlgAreaID}=108 \&$ subsecid $=900003$. Accessed 26 Dec 2010

24. Pickert K (2010) The unsustainable U.S. health care system. Time magazine. Available via http://swampland.blogs.time.com/2010/ 02/04/the-unsustainable-u-s-health-care-system/. Accessed 26 Dec 2010

25. Beinfeld MT, Gazelle GS (2005) Diagnostic imaging costs: are they driving up the costs of hospital care? Radiology 235:934-939

26. Chernew M, Royalty AB, Kronick R et al (2009) Geographic variation and health care cost growth: research to inform a complex diagnosis. Robert Wood Johnson Foundation. Available via http:// www.rwjf.org/pr/product.jsp?id=50888. Accessed 26 Dec 2010

27. Saif MW, Tzannou I, Makrilla N et al (2010) Role and cost effectiveness of PET/CT in management of patients with cancer. Yale J Biol Med 83:53-65

28. Stoker J, van Randen A, Lameris W et al (2009) Imaging patients with acute abdominal pain. Radiology 253:31-46

29. Schmidt T, Muhlberger N, Chemelli-Steingruber IE et al (2010) Benefit, risks, and cost-effectiveness of screening for abdominal aortic aneurysm. Rofo 182:573-580

30. Hendee WR, Becker GJ, Borgstede JP et al (2010) Addressing overutilization in medical imaging. Radiology 257:240-245

31. Chawla A, Gunderman RB (2008) Defensive medicine: prevalence, implications, and recommendations. Acad Radiol 15:948-949

32. Dumitriu D, Galloy MA, Claudon M (2010) New techniques in pediatric ultrasound. Ultrasound Clin 5:153-169

33. Kavanagh EC, Koulouris G, Parker L et al (2008) Does extendedfield-of-view sonography improve interrater reliability for the detection of rotator cuff muscle atrophy? AJR 190:27-31 
34. Yerli H, Eksioglu SY (2009) Extended field-of-view sonography: evaluation of the superficial lesions. Can Assoc Radiol J 60:35-39

35. Nelson TR, Downey DB, Pretorius DH et al (1999) Threedimensional ultrasound. Lippincott Williams \& Wilkins, Philadelphia

36. Benacerraf BR, Shipp TD, Bromley B (2009) Three-dimensional ultrasound detection of abnormally located intrauterine contraceptive devices which are a source of pelvic pain and abnormal bleeding. Ultrasound Obstet Gynecol 34:110-115

37. Tache V, Tarsa M, Romine L et al (2008) Three-dimensional obstetric ultrasound. Semin Ultrasound CT MR 29:147-155

38. Csutak R, Unterassinger L, Rohrmeister C et al (2003) Threedimensional volume measurement of the lateral ventricles in preterm and term infants: evaluation of a standardised computerassisted method in vivo. Pediatr Radiol 33:104-109

39. Riccabona M (2005) Pediatric three-dimensional ultrasound: basics and potential clinical value. Clin Imaging 29:1-5

40. Riccabona M, Fritz G, Ring E (2003) Potential applications of three-dimensional ultrasound in the pediatric urinary tract: pictorial demonstration based on preliminary results. Eur Radiol 13:2680-2687

41. Fordham LA (2004) Congenital abnormalities of the musculoskeletal system: perinatal evaluation and long-term outcome. Semin Roentgenol 39:304-322

42. Benacerraf BR, Shipp TD, Bromley B (2006) Three-dimensional US of the fetus: volume imaging. Radiology 238:988-996

43. Bromley B, Shipp TD, Benacerraf B (2007) Assessment of the third-trimester fetus using 3-dimensional volumes: a pilot study. J Clin Ultrasound 35:231-237

44. Nelson TR, Pretorius DH, Lev-Toaff A et al (2001) Feasibility of performing a virtual patient examination using three-dimensional ultrasonographic data acquired at remote locations. J Ultrasound Med 20:941-952

45. Miller DL, Averkiou MA, Brayman AA et al (2008) Bioeffects considerations for diagnostic ultrasound contrast agents. J Ultrasound Med 27:611-632

46. Addelmoneim SS, Bernier M, Scott CG et al (2009) Safety of contrast agent use during stress echocardiography: a 4-year experience from a single-center cohort study of 26, 774 patients. JACC Cardiovasc Imaging 2:1048-1056

47. Piscaglia F, Lencionai R, Sagrini E et al (2010) Characterization of focal liver lesions with contrast-enhanced ultrasound. Ultrasound Med Biol 36:531-550

48. Wilson SR, Burns PN (2010) Microbubble-enhanced US in body imaging: what role? Radiology 257:24-39

49. Darge K (2010) Voiding urosonography with US contrast agent for the diagnosis of vesicoureteric reflux in children: an update. Pediatr Radiol 40:956-962
50. Gessner R, Dayton PA (2010) Advances in molecular imaging with ultrasound. Molec Imaging 9:117-127

51. Hahn MA, Singh AK, Sharma P et al (2010) Nanoparticles as contrast agents for in-vivo bioimaging: current status and future perspectives. Anal Bioanal Chem 399:3-27

52. Wirtzfeld LA, Ghoshal G, Hafez ZT et al (2010) Cross-imaging platform comparison of ultrasonic backscatter coefficient measurements of live rat tumors. J Ultrasound Med 29:1117-1123

53. Wells PN (2006) Ultrasound imaging. Phys Med Biol 51:R83-98

54. Konofagou EE (2004) Quo vadis elasticity imaging? Ultrasonics 42:331-336

55. Bojunga J, Herrmann E, Meyer G et al (2010) Real-time elastography for the differentiation of benign and malignant thyroid nodules: a meta-analysis. Thyroid 20:1145-1150

56. Adamietz BR, Meier-Meitinger M, Fasching P et al (2010) New diagnostic criteria in real-time elastography for the assessment of breast lesions. Ultraschall Med PMID21165816 Dec 16 Epub ahead of print

57. Gheorghe L, Iacob S, Gheorghe C (2008) Real-time sonoelastography - a new application in the field of liver disease. J Gastrointestin Liver Dis 17:469-474

58. Carstensen EL, Parker KJ, Lerner RM (2008) Elastography in the management of liver disease. Ultrasound Med Biol 34:1535-1546

59. Vizzutti F, Arena U, Nobili V et al (2009) Non-invasive assessment of fibrosis in non-alcoholic fatty liver disease. Ann Hepatol 8:89-94

60. Pariente D, Franchi-Abella S (2010) Paediatric chronic liver diseases: how to investigate and follow up? Role of imaging in the diagnosis of fibrosis. Pediatr Radiol 40:906-919

61. Agdi M, Tulandi T (2010) Minimally invasive approach for myomectomy. Semin Reprod Med 28:228-234

62. McWilliams JP, Yamamoto S, Raman SS et al (2010) Percutaneous ablation of hepatocellular carcinoma: current status. J Vasc Interv Radiol 21:S204-213

63. Rove KO, Sullivan KF, Crawford ED (2010) High-intensity focused ultrasound: ready for primetime. Urol Clin North Am $37: 27-35$

64. Li C, Wu P, Zhang L et al (2009) Osteosarcoma: limb salvage treatment by ultrasonographically guided high-intensity focused ultrasound. Cancer Biol Ther 8:1102-1108

65. Vaezy S, Zderic V (2007) Hemorrhage control using high intensity focused ultrasound. Int J Hyperthermia 23:203-211

66. Hitchcock KE, Holland CK (2010) Ultrasound-assisted thrombolysis for stroke therapy: better thrombus break-up with bubbles. Stroke 41:S50-53

67. Stride EP, Coussios CC (2010) Cavitation and contrast: the use of bubbles in ultrasound imaging and therapy. Proc Inst Mech Eng $\mathrm{H}$ 224:171-191

68. Yoon CS, Park JH (2010) Ultrasound-mediated gene delivery. Expert Opin Drug Deliv 7:321-330 